TRANSACTIONS OF THE

AMERICAN MATHEMATICAL SOCIETY

Volume 352, Number 6, Pages 2553-2568

S 0002-9947(00)02619-2

Article electronically published on February 14, 2000

\title{
THETA LINE BUNDLES AND THE DETERMINANT OF THE HODGE BUNDLE
}

\author{
ALEXIS KOUVIDAKIS
}

\begin{abstract}
We give an expression of the determinant of the push forward of a symmetric line bundle on a complex abelian fibration, in terms of the pull back of the relative dualizing sheaf via the zero section.
\end{abstract}

\section{INTRODUCTION}

Let $\mathrm{f}: X \longrightarrow B$ be a fibration of abelian varieties with a zero section $s: B \longrightarrow X$. Let $\mathcal{L}$ be a symmetric line bundle on $X$, trivialized along the zero section, which defines a polarization of type $D=\left(d_{1}, \ldots, d_{g}\right)$ on the fibration. A theorem of Faltings and Chai (4, Ch. 1, Theorem 5.1) states that $8 d^{3} \operatorname{det}_{*} \mathcal{L}=-4 d^{4} s^{*} \omega_{X / B}$, where $\omega_{X / B}$ is the relative dualizing sheaf of the fibration and $d:=d_{1} \cdots d_{g}$. In this note we show that in the complex analytic category, the above torsion factor can be improved. More specifically, we have

Theorem A. Let $\mathrm{f}: X \longrightarrow B$ be a fibration of complex abelian varieties of relative dimension $g$, and let $s$ be the zero section. Let $\mathcal{L}$ be a symmetric line bundle on $X$, trivialized along the zero section, which defines a polarization of type $D=\left(d_{1}, \ldots, d_{g}\right)$, where $d_{1}|\cdots| d_{g}$ are positive integers. Let $d=d_{1} \cdots d_{g}$. Then $8 \operatorname{det}_{*} \mathcal{L}=-4 d s^{*} \omega_{X / B}$, except when $3 \mid d_{g}$ and $\operatorname{gcd}\left(3, d_{g-1}\right)=1$, in which case $24 \operatorname{det} \mathrm{f}_{*} \mathcal{L}=-12 d s^{*} \omega_{X / B}$.

Moreover, when $\mathcal{L}$ is totally symmetric (and therefore $d$ is an even integer), we have

Theorem B. Keeping the notation of Theorem $\mathbb{A}$, assume in addition that $\mathcal{L}$ is a totally symmetric line bundle on $X$ and that $g \geq 3$. Then $\operatorname{det}_{*} \mathcal{L}=-\frac{d}{2} s^{*} \omega_{X / B}$, except when $3 \mid d_{g}$ and $\operatorname{gcd}\left(3, d_{g-1}\right)=1$, in which case $3 \operatorname{det} f_{*} \mathcal{L}=-3 \frac{d}{2} s^{*} \omega_{X / B}$.

The theorems are proved by using a refinement of the theta transformation formula, see Propositions 2.1 and 2.2 in order to construct transition functions for $\operatorname{det} f_{*}(\mathcal{L})$, see Lemma 3.1 .

In the last section, we apply Theorem B to the case of the universal Jacobian variety $\mathrm{f}_{g-1}: \mathcal{J}^{g-1} \longrightarrow \mathcal{M}_{g}$, where $\mathcal{M}_{g}$ denotes the moduli space of smooth, irreducible curves of genus $g \geq 3$, without automorphisms. This is an abelian torsor which parametrizes line bundles of degree $g-1$ on the fibers of the universal curve $\psi: \mathcal{C} \longrightarrow \mathcal{M}_{g}$. On $\mathcal{J}^{g-1}$, there is a canonical theta divisor defined as the push forward of the universal symmetric product of degree $g-1$, via the Abel-Jacobi

Received by the editors June 1, 1997.

2000 Mathematics Subject Classification. Primary 14D05, 14K25, 14L35; Secondary 11 F03.

(C)2000 American Mathematical Society 
map. We denote by $\Theta$ the corresponding line bundle and let $\lambda=\operatorname{det} \psi_{*} \omega_{\mathcal{C} / \mathcal{M}_{g}}$ be the determinant of the Hodge bundle. We then have

Theorem C. In the above notation, $\operatorname{det} \mathrm{f}_{g-1 *}\left(\Theta^{\otimes n}\right)=\frac{1}{2} n^{g}(n-1) \lambda$.

We also give an alternative way for proving Theorem C by utilizing special properties of the universal Jacobian varieties.

\section{ACKNOWLEDGMENT}

I would like to thank Professor L. Moret-Bailly for showing me the alternative way for proving Theorem $\mathrm{C}$.

\section{Abelian VARIEties AND theta FunCtions}

We recall in this section some standard theory for complex abelian varieties and theta functions. We follow the book by Lange and Birkenhake [5]. We denote by $X=V / \Lambda$ an abelian variety; $V$ is a $\mathbb{C}$-vector space of dimension $g$ and $\Lambda$ a $2 g$-lattice of maximal rank in $V$.

Line bundles on abelian varieties. A line bundle on $X$ is determined, up to isomorphism, by an hermitian form $H$ on $V$ such that $\operatorname{Im} H(\Lambda, \Lambda) \subseteq \mathbb{Z}$, and by a semicharacter $\chi: \Lambda \longrightarrow \mathbb{C}_{1}([5]$, Ch. $2, \S 2)$. We denote by $L(H, \chi)$ a line bundle, up to isomorphism, given by the above data. If $\phi: X^{\prime}=V^{\prime} / \Lambda^{\prime} \longrightarrow X=V / \Lambda$ is a map of abelian varieties, we denote by $\phi_{a}: V^{\prime} \longrightarrow V$ and $\phi_{r}: \Lambda^{\prime} \longrightarrow \Lambda$ the analytic and the rational representation of $\phi$ respectively $([5]$, Ch. $1, \S 2)$. Given $L(H, \chi)$ on $X$, we have that $\phi^{*} L(H, \chi)=L\left(\phi_{a}^{*} H, \phi_{r}^{*} \chi\right)$.

Let $\mathcal{B}^{s}$ be the symplectic base of $\Lambda$ w.r.t. which the alternating form $E:=\operatorname{Im} H$ is represented by a matrix $\left(\begin{array}{cc}0 & D \\ -D & 0\end{array}\right)$, where $D$, the polarization type, is an integral diagonal matrix with elements $d_{1}|\ldots| d_{g}$. Let $\Lambda_{1}$ (resp. $\Lambda_{2}$ ) be the lattice spanned by the first (resp. last) $g$ vectors of $\mathcal{B}^{s}$. Then $\Lambda=\Lambda_{1} \oplus \Lambda_{2}$, and we write $\lambda=\lambda_{1}+\lambda_{2}$ for the corresponding decomposition of $\lambda \in \Lambda$. This induces a decomposition $V=$ $V_{1} \oplus V_{2}$, where $V_{i}=\Lambda_{i} \otimes \mathbb{R}$, which is called decomposition of $V$ for $H$. If $v \in V$, we write $v=v_{1}+v_{2} \in V_{1} \oplus V_{2}$. We define $\Lambda(H):=\{v \in V: \operatorname{Im} H(v, \Lambda) \subset \mathbb{Z}\}$. Then $\Lambda(H)=\Lambda(H)_{1} \oplus \Lambda(H)_{2}$, where $\Lambda(H)_{i}:=\Lambda(H) \cap V_{i}$.

We choose a decomposition of $V$ for $H$. Then we can define a distinguished line bundle $L\left(H, \chi_{0}\right)$, by setting $\chi_{0}(\lambda)=e\left(\pi i E\left(\lambda_{1}, \lambda_{2}\right)\right)$. A characteristic of a line bundle $L(H, \chi)$ is an element $c \in V$, unique up to translations by elements of $\Lambda(H)$, determined by the property $L(H, \chi)=T_{c}^{*} L\left(H, \chi_{0}\right)$, where $T_{c}$ is the translation by $c$ (see [5, Ch. 3, §1).

Period matrices. Let $\mathfrak{h}_{g}$ denote the Siegel upper half space of dimension $g$. We fix a polarization type $D$. A matrix $Z \in \mathfrak{h}_{g}$ determines a triple $\left(X_{Z}, H_{Z}, \mathcal{B}_{Z}^{s}\right)$, where $X_{Z}:=\mathbb{C}^{g} / \Lambda_{Z}$ (with $\Lambda_{Z}:=(Z, D) \mathbb{Z}^{2 g}$ ) is an abelian variety, $H_{Z}$ is an hermitian form of type $D$ with matrix $(\operatorname{Im} Z)^{-1}$ w.r.t. the standard base of $\mathbb{C}^{g}$, and $\mathcal{B}^{s}$ is the symplectic base spanned by the column vectors of the matrix $(Z, D)$; see [5], Ch. 8 , $\S 1$. 
THETA LINE BUNDLES AND THE DETERMINANT OF THE HODGE BUNDLE 2555

Canonical-classical factor of automorphy. A factor of automorphy is a holomorphic map $f: \Lambda \times V \longrightarrow \mathbb{C}^{\times}$satisfying

$$
f\left(\lambda_{1}+\lambda_{2}, v\right)=f\left(\lambda_{1}, \lambda_{2}+v\right) f\left(\lambda_{2}, v\right) .
$$

Two factors of automorphy $f$ and $f^{\prime}$ are called equivalent if

$$
f^{\prime}(\lambda, v)=f(\lambda, v) h(v) h(v+\lambda)^{-1}
$$

for some holomorphic function $h: V \rightarrow \mathbb{C}^{\times}$. We use the notation $f^{\prime}=f \star h$ for this situation.

Given an hermitian form $H$, we denote by $B$ the symmetric form on $V$ associated to $H$ ([5], Ch.3, Lemma 2.1). Given data $(H, \chi)$, we define by $a_{(H, \chi)}(\lambda, v):=$ $\chi(\lambda) e\left(\pi H(v, \lambda)+\frac{\pi}{2} H(\lambda, \lambda)\right)$ the canonical factor of automorphy and by $e_{(H, \chi)}(\lambda, v)$ $:=\chi(\lambda) e\left(\pi(H-B)(v, \lambda)+\frac{\pi}{2}(H-B)(\lambda, \lambda)\right)$ the classical factor of automorphy. When the semicharacter is $\chi_{0}$, we simplify the notation for the above factors of automorphy to $a_{H}$ and $e_{H}$.

Canonical-classical theta functions. A theta function corresponding to a factor of automorphy $f$ is a holomorphic function $\theta: V \longrightarrow \mathbb{C}$ satisfying the functional equation $\theta(\lambda+v)=f(\lambda, v) \theta(v)$. Theta functions corresponding to the canonical (resp. classical) factor of automorphy $a_{(H, \chi)}$ (resp. $\left.e_{(H, \chi)}\right)$ are called canonical (resp. classical) theta functions for $L(H, \chi)$. Let $c$ be a characteristic of $L(H, \chi)$. We define

$$
\begin{aligned}
\theta^{c}(v):=e( & \left.-\pi H(v, c)-\frac{\pi}{2} H(c, c)+\frac{\pi}{2} B(v+c, v+c)\right) \\
& \cdot \sum_{\lambda_{1} \in \Lambda_{1}} e\left(\pi(H-B)\left(v+c, \lambda_{1}\right)-\frac{\pi}{2}(H-B)\left(\lambda_{1}, \lambda_{1}\right)\right) .
\end{aligned}
$$

We have the following ([5], Ch. $3, \S \S 1$ and 2$)$ :

i) $\theta^{c}$ is a canonical theta function and $\theta^{c}(v)=e\left(-\pi H(v, c)-\frac{\pi}{2} H(c, c)\right) \theta(v+c)$, where $\theta:=\theta^{0}$.

ii) Let $\theta_{\bar{w}}^{c}(v):=a_{(H, \chi)}(w, v)^{-1} \theta^{c}(v+w)$, where $\bar{w} \in K(H):=\Lambda(H) / \Lambda$. The set $\left\langle\theta_{w}^{c}: \bar{w} \in K(H)_{1}:=\Lambda(H)_{1} / \Lambda_{1}\right\rangle$ forms a base of the canonical theta functions.

Let $Z \in \mathfrak{h}_{g}$ and let $D$ be a fixed polarization type. Let $X_{Z}:=\mathbb{C}^{g} / \Lambda_{Z}$ be the abelian variety corresponding to $Z$ and let $H=H_{Z}$. Given $v \in \mathbb{C}^{g}$, we can write uniquely $v=Z v^{1}+v^{2}$, where $v^{i} \in \mathbb{R}^{g}$. If $\lambda \in \Lambda_{Z}$ then it can be written uniquely in the form $\lambda=Z \lambda^{1}+\lambda^{2}$, where $\lambda^{1} \in \mathbb{Z}^{g}$ and $\lambda^{2} \in D \mathbb{Z}^{g}$. Let $L(H, \chi)$ be a line bundle on $X_{Z}$ of characteristic $c$ w.r.t. the natural decomposition of $\mathbb{C}^{g}$ determined by $Z$. We have the following (many of them can be found in [5], Ch. $8, \S 5$; the rest is a straightforward calculation):

1. $H(v, w)={ }^{t} v(\operatorname{Im} Z)^{-1} \bar{w}, B(v, w)={ }^{t} v(\operatorname{Im} Z)^{-1} w$. $(H-B)(v, w)=-2 i^{t} v w^{1}, \quad E(v, w)={ }^{t} v^{1} w^{2}-{ }^{t} v^{2} w^{1}$.

2. $e_{(H, \chi)}(\lambda, v)=e\left(2 \pi i\left({ }^{t} c^{1} \lambda^{2}-{ }^{t} c^{2} \lambda^{1}\right)-\pi i^{t} \lambda^{1} Z \lambda^{1}-2 \pi i^{t} v \lambda^{1}\right)$. Also,

$a_{(H, \chi)}(\lambda, v)=e\left(\pi i^{t} \lambda^{1} \lambda^{2}+2 \pi i\left({ }^{t} c^{1} \lambda^{2}-{ }^{t} c^{2} \lambda^{1}\right)+\pi{ }^{t} v(\operatorname{Im} Z)^{-1} \bar{\lambda}+\frac{\pi}{2}{ }^{t} \lambda(\operatorname{Im} Z)^{-1} \bar{\lambda}\right)$.

It is $e_{(H, \chi)}=a_{(H, \chi)} \star h$, where $h(v)=e\left(\frac{\pi}{2}^{t} v(\operatorname{Im} Z)^{-1} v\right)$.

3. Let $\mathbb{Z}_{D}$ denote the group $\mathbb{Z}_{D}:=\mathbb{Z}_{d_{1}} \oplus \cdots \oplus \mathbb{Z}_{d_{g}}$. Then $\Lambda(H)_{1}=\left\{Z v^{1} \mid v^{1} \in\right.$ $\left.D^{-1} \mathbb{Z}^{g}\right\}, \quad \Lambda(H)_{2}=\left\{v^{2} \mid v^{2} \in \mathbb{Z}^{g}\right\}$ and $K(H)_{1} \cong D^{-1} \mathbb{Z}_{D}, \quad K(H)_{2} \cong \mathbb{Z}_{D}$. 
4. Let $c=Z c^{1}+c^{2}$. Then

$$
\theta\left[\begin{array}{l}
c^{1} \\
c^{2}
\end{array}\right](v, Z):=e\left(-\frac{\pi}{2} B(v, v)+\pi i^{t} c^{1} c^{2}\right) \theta^{c}(v)
$$

is a classical theta function and

$$
\theta\left[\begin{array}{l}
c^{1} \\
c^{2}
\end{array}\right](v, Z)=\sum_{\lambda^{1} \in \mathbb{Z}^{g}} e\left(\pi i^{t}\left(\lambda^{1}+c^{1}\right) Z\left(\lambda^{1}+c^{1}\right)+2 \pi i^{t}\left(v+c^{2}\right)\left(\lambda^{1}+c^{1}\right)\right) .
$$

The set

$$
\left\langle\theta\left[\begin{array}{c}
c^{1}+D^{-1} m \\
c^{2}
\end{array}\right], m \in \mathbb{Z}_{D}\right\rangle
$$

forms a base of the classical theta functions.

5. Let $c=Z c^{1}+c^{2} \in \mathbb{C}^{g}, w=Z w^{1}+w^{2} \in \Lambda(H)$ and $Z s^{1} \in \Lambda(H)_{1}$. Then:
a) $\theta\left[\begin{array}{c}c^{1}+w^{1} \\ c^{2}\end{array}\right](v, Z)=e\left(-\frac{\pi}{2} B(v, v)+\pi i^{t} c^{1} c^{2}\right) \theta \frac{c}{w}(v)$.
b) $\theta\left[\begin{array}{l}c^{1}+w^{1} \\ c^{2}+w^{2}\end{array}\right](v, Z)=e\left(2 \pi i^{t}\left(c^{1}+w^{1}\right) w^{2}\right) \theta\left[\begin{array}{c}c^{1}+w^{1} \\ c^{2}\end{array}\right](v, Z)$.
c) $\theta \frac{c}{Z w^{1}+w^{2}}(v)=\theta \frac{c}{Z w^{1}}(v)$.
d) $\theta^{c+w}(v)=e\left(-\pi i^{t} c^{2} w^{1}+\pi i^{t}\left(c^{1}+w^{1}\right) w^{2}\right) \theta \frac{c}{w}(v)$.
e) $\theta \frac{c+Z s^{1}}{Z w^{1}}(v)=e\left(-\pi i^{t} s^{1} c^{2}\right) \theta \frac{c}{Z\left(w^{1}+s^{1}\right)}(v)$.

Action of the symplectic group. Let $D$ be a polarization type and let $A_{D}:=$ $\left(\begin{array}{cc}0 & D \\ -D & 0\end{array}\right)$ and $I_{D}:=\left(\begin{array}{cc}I & 0 \\ 0 & D\end{array}\right)$. We set $\Gamma_{D}:=\left\{R \in M_{2 g}(\mathbb{Z}), R A_{D}{ }^{t} R=\right.$ $\left.A_{D}\right\}$ and $G_{D}:=\left\{M \in \operatorname{Sp}_{2 g}(\mathbb{Q}), M=I_{D}^{-1} R I_{D}\right.$, for some $\left.R \in \Gamma_{D}\right\}$. If $R=$ $\left(\begin{array}{cc}A & B \\ \Gamma & \Delta\end{array}\right) \in \Gamma_{D}$ and $M=\left(\begin{array}{cc}\alpha & \beta \\ \gamma & \delta\end{array}\right) \in G_{D}$, then $\alpha=A, \beta=B D, \gamma=D^{-1} \Gamma$, $\delta=D^{-1} \Delta D$. We have $A D^{t} \Delta-B D^{t} \Gamma=D$. Also, the matrices $\Gamma D^{t} \Delta, A D^{t} B$, ${ }^{t} A D^{-1} \Gamma,{ }^{t} \Delta D^{-1} B$ are symmetric and the matrices $D^{t} A D^{-1}, D^{t} B D^{-1}, D^{t} \Gamma D^{-1}$, $D^{t} \Delta D^{-1}$ are integral.

The group $G_{D}$ acts on $\mathfrak{h}_{g}$ by $M(Z):=(\alpha Z+\beta)(\gamma Z+\delta)^{-1}$ ([5], Ch. 8, $\left.\S 1\right)$. Two abelian varieties $X_{Z}$ and $X_{Z^{\prime}}$ of type $D$ are isomorphic if $Z^{\prime}=M(Z)$. The isomorphism is given by $\phi(M): X_{Z} \longrightarrow X_{M(Z)}$, so that the corresponding map $\phi(M)_{r}: \Lambda_{Z} \longrightarrow \Lambda_{M(Z)}$ has matrix $R={ }^{t} M^{-1}$ w.r.t. the canonical symplectic bases defined by $Z$ and $M(Z)$. Let $j_{Z}: \mathbb{R}^{2 g} \longrightarrow \mathbb{C}^{g}$ be the isomorphism given by $x \mapsto(Z, 1) x$. We have the following diagram $([5]$, Ch. $8, \S 8)$ :

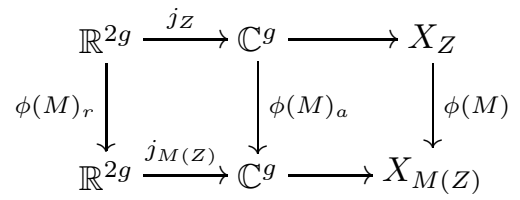

The map $\phi(M)_{a}: \mathbb{C}^{g} \longrightarrow \mathbb{C}^{g}$ has matrix $A^{-1}$, where $A={ }^{t}(\gamma Z+\delta)$, w.r.t. the standard base of $\mathbb{C}^{g}$. Moreover, $\phi(M)_{a}^{*} H_{M(Z)}=H_{Z}$. We define $M_{Z}(v):=A^{-1} v(=$ $\left.\phi(M)_{a}(v)\right)$. 
THETA LINE BUNDLES AND THE DETERMINANT OF THE HODGE BUNDLE 2557

Factors of automorphy and line bundles. Sections and theta functions. A factor of automorphy $f: \Lambda \times V \rightarrow \mathbb{C}^{\times}$defines an action of $\Lambda$ on $V \times \mathbb{C}$ given by $\lambda(v, z):=(v+\lambda, f(\lambda, v) z)$. The quotient of $V \times \mathbb{C}$ by this action defines a line bundle $L$ on $X$, the elements of which we denote by $[v, z]$. If $f^{\prime}=f \star h$, then for the corresponding line bundles $L$ and $L^{\prime}$ there exists a canonical isomorphism $\Phi_{h}: L \longrightarrow L^{\prime}$ given by $[v, z] \mapsto\left[v, h(v)^{-1} z\right]$. Given a map of abelian varieties $\phi: X^{\prime} \longrightarrow X$, we define $\phi^{*} f:=\left(\phi_{r} \times \phi_{a}\right)^{*} f$, which is a factor of automorphy for $X^{\prime}$. Then $\phi^{*} L$ is the line bundle on $X^{\prime}$ corresponding to $\phi^{*} f$. If $\theta$ is a theta function for $f$, then $\phi_{a}^{*} \theta$ (or $\phi^{*} \theta$ in a simplified notation) is a theta function for $\phi^{*} f$.

Sections of the line bundle $L$ correspond to theta functions $\theta: V \rightarrow \mathbb{C}$ satisfying the functional equation $\theta(\lambda+v)=f(\lambda, v) \theta(v)$. The relation is the following. Given a section $s$ of $L$, let $s(x)=\left[v_{s}(x), z_{s}(x)\right]$. We then define $\theta_{s}(v):=$ $f\left(v-v_{s}(x), v_{s}(x)\right) z_{s}(x)$. Conversely, given a theta function $\theta$ for $f$, we define $s(x):=[v(x), \theta(v(x))]$, where $v(x)$ is an arbitrary vector which lies over $x$. If $\phi:$ $X^{\prime} \longrightarrow X$ is a map as above and $s \in H^{0}(X, L)$ is a section corresponding to $\theta$, then the section $\phi^{*} s \in H^{0}\left(X^{\prime}, \phi^{*} L\right)$ corresponds to $\phi^{*} \theta$. Suppose $f^{\prime}=f \star h$. Then given a section $s^{\prime} \in H^{0}\left(X, L^{\prime}\right)$ corresponding to $\theta_{s^{\prime}}$, we have that $s:=\Phi_{h}^{*} s^{\prime} \in H^{0}(X, L)$ corresponds to $\theta_{s}:=h(v) \theta_{f^{\prime}}(v)$.

\section{THETA TRANSFORMATION FORMULA}

Let $Z \in \mathfrak{h}_{g}$, and let $L\left(H_{Z}, \chi\right)$ be a line bundle of characteristic $c$ on the abelian variety $X_{Z}$. Let $M \in G_{D}$ and define $Z^{\prime}:=M(Z)$ as in Section 1, Let $\psi=\psi(M)$ : $X_{Z^{\prime}} \longrightarrow X_{Z}$ be the inverse of the map $\phi=\phi(M): X_{Z} \longrightarrow X_{Z^{\prime}}$. The line bundle $\psi^{*} L\left(H_{Z}, \chi\right)$ has type $\psi^{*} H_{Z}=H_{Z^{\prime}}$, semicharacter $\chi^{\prime}=\psi^{*} \chi$ and characteristic $M[c]$, with $M[c]^{1}=\delta c^{1}-\gamma c^{2}+\frac{1}{2}\left(D \gamma^{t} \delta\right)_{0}$ and $M[c]^{2}=-\beta c^{1}+\alpha c^{2}+\frac{1}{2}\left(\alpha^{t} \beta\right)_{0}$ (see [5], Ch. 8, Lemma 4.1, where there is an unfortunate omission of $D$ in the expression of $\left.M[c]^{1}\right)$. (The ( )o stands for the diagonal vector.)

Lemma 2.1. We have that

$$
\psi^{*} e_{\left(H_{Z}, \chi\right)}=e_{\left(H_{Z^{\prime}}, \chi^{\prime}\right)} \star h^{\prime},
$$

where $h^{\prime}(v)=e\left(\pi i^{t} v(\gamma Z+\delta)^{-1} \gamma v\right)$. Also,

$$
\phi^{*} e_{\left(H_{Z^{\prime}}, \chi^{\prime}\right)}=e_{\left(H_{Z}, \chi\right)} \star h,
$$

where $h(v)=e\left(-\pi i^{t} v(\gamma Z+\delta)^{-1} \gamma v\right)$.

Proof. We have that $a_{\left(H_{Z}, \chi\right)}=e_{\left(H_{Z}, \chi\right)} \star h_{1}$, where $h_{1}(v)=e\left(-\frac{\pi}{2}^{t} v(\operatorname{Im} Z)^{-1} v\right)$ (see item 2 in Section 11). Since $\psi^{*} a_{\left(H_{Z}, \chi\right)}=a_{\left(H_{Z^{\prime}}, \chi^{\prime}\right)}$ and $a_{\left(H_{Z^{\prime}}, \chi^{\prime}\right)}=e_{\left(H_{Z^{\prime}}, \chi^{\prime}\right)}$ * $h_{1}^{\prime}$, where $h_{1}^{\prime}\left(v^{\prime}\right)=e\left(-\frac{\pi}{2}^{t} v^{\prime}\left(\operatorname{Im} Z^{\prime}\right)^{-1} v^{\prime}\right)$, by applying $\psi^{*}$ we get that $h^{\prime}(v)=$ $\psi^{*} h_{1}(v)^{-1} h_{1}^{\prime}\left(v^{\prime}\right)$, i.e., $h^{\prime}(v)=e\left(\frac{\pi}{2}^{t} v(\operatorname{Im} Z)^{-1} v-\frac{\pi}{2}^{t} v^{\prime}\left(\operatorname{Im} Z^{\prime}\right)^{-1} v^{\prime}\right)$, where $v^{\prime}:=$ $\phi_{a}(v)$. A straightforward calculation gives the above form for $h^{\prime}$. To prove the second formula, we apply $\phi^{*}$ to the first one.

The tuple $B^{Z}:=\left\langle\theta \frac{c}{Z D^{-1} m}(v) ; m \in \mathbb{Z}_{D}\right\rangle$ forms a base of the canonical theta functions for $L\left(H_{Z}, \chi\right)$ and the tuple $B^{Z^{\prime}}:=\left\langle\theta \frac{M[c]}{Z^{\prime} D^{-1} n}\left(v^{\prime}\right) ; n \in \mathbb{Z}_{D}\right\rangle$ forms a base of the canonical theta functions for $L\left(H_{Z^{\prime}}, \chi^{\prime}\right)$. On the other hand, the tuple $\psi^{*} B^{Z}:=\left\langle\psi_{a}^{*} \theta \frac{c}{D^{-1} m}(v) ; m \in \mathbb{Z}_{D}\right\rangle$ also forms a base of the canonical theta functions for $L\left(H_{Z^{\prime}}, \chi^{\prime}\right)$, since $\psi^{*} L\left(H_{Z}, \chi\right)=L\left(H_{Z^{\prime}}, \chi^{\prime}\right)$ and $\psi^{*} a_{\left(H_{Z}, \chi\right)}=a_{\left(H_{Z^{\prime}}, \chi^{\prime}\right)}$. 
Proposition 2.1. Keeping the above notation, assume that the characteristic $c \in$ $\frac{1}{2} \Lambda\left(H_{Z}\right)$. Then the matrix $C$, for which $\psi^{*} B^{Z}=C B^{Z^{\prime}}$, is of the form $C=$ $(\operatorname{det}(\gamma Z+\delta))^{-\frac{1}{2}} C(M)$, where $C(M)$ depends on $M$ and $\operatorname{det} C(M)=\zeta_{8}$, except when $3 \mid d_{g}$ and $\left(d_{g-1}, 3\right)=1$, in which case we have $\operatorname{det} C(M)=\zeta_{24}$ (by $\zeta_{m}$ we denote an m-root of unity).

Proof. Let $G_{D}^{\text {int }}:=G_{D} \cap \mathrm{Sp}_{2 g}(\mathbb{Z})$. A matrix $M$ belongs to $G_{D}^{\text {int }}$ if

$$
M=\left(\begin{array}{cc}
I & 0 \\
0 & D
\end{array}\right)^{-1} R\left(\begin{array}{cc}
I & 0 \\
0 & D
\end{array}\right)
$$

where $R=\left(\begin{array}{cc}A & B \\ \Gamma & \Delta\end{array}\right) \in \Gamma_{D}$ and $\Gamma=D \Gamma_{1}, \Gamma_{1} \in M_{g}(\mathbb{Z})$. Therefore, define $\Gamma_{D}^{\text {int }}:=\left\{R=\left(\begin{array}{cc}A & B \\ \Gamma & \Delta\end{array}\right) \in \Gamma_{D}\right.$, where $\left.\Gamma=D \Gamma_{1}, \Gamma_{1} \in M_{g}(\mathbb{Z})\right\}$. We have the following lemma:

Lemma 2.2. The group $\Gamma_{D}$ is generated by $\Gamma_{D}^{\mathrm{int}}$ and the matrix $J:=\left(\begin{array}{cc}0 & -I \\ I & 0\end{array}\right)$, and so the group $G_{D}$ is generated by $G_{D}^{\mathrm{int}}$ and the matrix $\left(\begin{array}{cc}0 & -D \\ D^{-1} & 0\end{array}\right)$.

Proof. We use results from 3 . Let $K(D)=D^{-1} \mathbb{Z}_{D} \oplus \mathbb{Z}_{D}$. A matrix $R \in \Gamma_{D}$ acts on $K(D)$ by multiplication by $\left(\begin{array}{cc}I & 0 \\ 0 & D\end{array}\right){ }^{t} R\left(\begin{array}{cc}I & 0 \\ 0 & D\end{array}\right)^{-1}$. By identifying $K(D)$ with $\mathbb{Z}_{D} \oplus \mathbb{Z}_{D}$ via the isomorphism given by the matrix $\left(\begin{array}{cc}D & 0 \\ 0 & I\end{array}\right)$, the action of $R \in \Gamma_{D}$ on $K(D)$ is given by multiplication by $\bar{D}^{t} R \bar{D}^{-1}$, where $\bar{D}:=\left(\begin{array}{cc}D & 0 \\ 0 & D\end{array}\right)$. One can define on $K(D)$ an alternating form $e^{D}([\underline{5},[\underline{3})$, and the above action becomes a symplectic action. Let $\operatorname{Sp}(D)$ be the symplectic group of $K(D)$ with respect to $e^{D}$. We then have an exact sequence $0 \longrightarrow \Gamma_{D}(D) \longrightarrow \Gamma_{D} \stackrel{\pi}{\longrightarrow} \operatorname{Sp}(D) \longrightarrow 0$, where $\pi(R):=\bar{D}^{t} R \bar{D}^{-1}$ and $\Gamma_{D}(D):=\left\{R \in \Gamma_{D} \mid R=I+\bar{D} A, A \in M_{2 g}(\mathbb{Z})\right\}$. Note that $\Gamma_{D}(D) \subset \Gamma_{D}^{\text {int }}$. It suffices therefore to show that every element of $\operatorname{Sp}(D)$ has a lift to an element of $\Gamma_{D}$ which is a product of the matrix $J$ and elements of $\Gamma_{D}^{\text {int }}$.

Following the notation of [3], we have that $A \in L_{D}$ if $\bar{D}^{t} A \bar{D}^{-1} \in \Gamma_{D}$, where $L_{D}$ is defined in Section 2 of [3]. In [3], Theorem 2, it is shown that a matrix $A \in \operatorname{Sp}(D)$ has a lift $\tilde{A} \in L_{D}$ which satisfies a relation

$$
\left(\begin{array}{ll}
I & 0 \\
c_{1} & I
\end{array}\right)\left(\begin{array}{cc}
U_{1} & 0 \\
0 & U_{2}
\end{array}\right)\left(\begin{array}{cc}
a & D y \\
-D & d
\end{array}\right)\left(\begin{array}{cc}
I & b_{1} \\
0 & I
\end{array}\right) \tilde{A}=\left(\begin{array}{cc}
I & b_{2} \\
0 & I
\end{array}\right),
$$

where $y$ is an integral diagonal matrix and all the matrices belong to $L_{D}$. The inverse of a matrix $\left(\begin{array}{ll}a & b \\ c & d\end{array}\right)$ in $L_{D}$ belongs to $L_{D}$ and is given by

$$
\bar{D}\left(\begin{array}{cc}
{ }^{t} d & -{ }^{t} b \\
-{ }^{t} c & { }^{t} a
\end{array}\right) \bar{D}^{-1}
$$

Therefore $A$ has a lift $R$ in $\Gamma_{D}$ which is given by $R=\bar{D}^{t} \tilde{A} \bar{D}^{-1}$. But $\left(\begin{array}{ll}I & 0 \\ c & I\end{array}\right)=$ $J\left(\begin{array}{cc}-I & c \\ 0 & -I\end{array}\right) J$; hence $R=\left(J N_{1} J\right) N_{2} N_{3} N_{4}\left(J N_{5} J\right)$, where $N_{i} \in \Gamma_{D}^{\text {int }}$. 
THETA LINE BUNDLES AND THE DETERMINANT OF THE HODGE BUNDLE 2559

As in [7, Ch. II, $\S 5$, we can rewrite the formula we want to prove as

$$
\begin{aligned}
& \left\langle\theta \frac{c}{Z D^{-1} m}(v, Z) ; m \in \mathbb{Z}_{D}\right\rangle \sqrt{d v_{1} \wedge \ldots \wedge d v_{g}} \\
& \quad=C(M)\left\langle\theta \frac{M[c]}{Z^{\prime} D^{-1} n}\left(v^{\prime}, Z^{\prime}\right) ; n \in \mathbb{Z}_{D}\right\rangle \sqrt{d v_{1}^{\prime} \wedge \ldots \wedge d v_{g}^{\prime}},
\end{aligned}
$$

where $v^{\prime}={ }^{t}(\gamma Z+\delta)^{-1} v$ and $Z^{\prime}=(\alpha Z+\beta)(\gamma Z+\delta)^{-1}$. Note that if $c \in \frac{1}{2} \Lambda\left(H_{Z}\right)$, then $M[c] \in \frac{1}{2} \Lambda\left(H_{Z^{\prime}}\right)$. We observe that if the formula holds for $M_{1}, M_{2} \in G_{D}$, then it also holds for $M_{1} M_{2}$. It suffices therefore to verify the proposition for the generators. We express the relation $\psi^{*} B^{Z}=C B^{Z^{\prime}}$ in terms of classical theta functions, and, by using Lemma 2.1 we get

$$
\begin{gathered}
\left\langle\theta\left[\begin{array}{c}
c^{1}+D^{-1} m \\
c^{2}
\end{array}\right](v, Z) ; m \in \mathbb{Z}_{D}\right\rangle=e\left(-\pi i^{t} M[c]^{1} M[c]^{2}+\pi i^{t} c^{1} c^{2}\right) \\
\cdot e\left(-\pi i^{t} v(\gamma Z+\delta)^{-1} \gamma v\right)(\operatorname{det}(\gamma Z+\delta))^{-\frac{1}{2}} \\
\cdot C(M)\left\langle\theta\left[\begin{array}{c}
M[c]^{1}+D^{-1} n \\
M[c]^{2}
\end{array}\right]\left(v^{\prime}, Z^{\prime}\right) ; n \in \mathbb{Z}_{D}\right\rangle .
\end{gathered}
$$

Matrices of the form $\left(\begin{array}{cc}0 & -D \\ D^{-1} & 0\end{array}\right)$. In this case $e\left(-\pi i^{t} v(\gamma Z+\delta)^{-1} \gamma v\right)=$ $e\left(-\pi i^{t} v Z^{-1} v\right)$ and $\operatorname{det}(\gamma Z+\delta)=\frac{\operatorname{det} Z}{d}$. We also have $v^{\prime}=D Z^{-1} v, Z^{\prime}=-D Z^{-1} D$ and $M[c]^{1}=-D^{-1} c^{2}, M[c]^{2}=D c^{1}$. Relation (2) in this case becomes

$$
\begin{aligned}
& \left\langle\theta\left[\begin{array}{c}
c^{1}+D^{-1} m \\
c^{2}
\end{array}\right](v, Z) ; m \in \mathbb{Z}_{D}\right\rangle=e\left(-\pi i^{t} v Z^{-1} v\right) e\left(2 \pi i^{t} c^{1} c^{2}\right) \\
& \cdot\left(\frac{\operatorname{det} Z}{d}\right)^{-\frac{1}{2}} C(M)\left\langle\theta\left[\begin{array}{c}
-D^{-1} c^{2}+D^{-1} n \\
D c^{1}
\end{array}\right]\left(v^{\prime}, Z^{\prime}\right) ; n \in \mathbb{Z}_{D}\right\rangle .
\end{aligned}
$$

As in [7], we apply Fourier transform. Write

$$
\theta\left[\begin{array}{c}
c^{1}+D^{-1} m \\
c^{2}
\end{array}\right](v, Z)=\sum_{\lambda \in \mathbb{Z}^{g}} f(\lambda)
$$

where

$$
\begin{aligned}
f(x):=e\left(\pi i^{t}\left(x+c^{1}+D^{-1} m\right) Z\left(x+c^{1}+D^{-1} m\right)\right. & \\
& \left.+2 \pi i^{t}\left(v+c^{2}\right)\left(x+c^{1}+D^{-1} m\right)\right) .
\end{aligned}
$$

Let $\hat{f}(x):=\int_{\mathbb{R}^{g}} f(x) e\left(2 \pi i^{t} x \lambda\right) d x$. We then have

$$
\theta\left[\begin{array}{c}
c^{1}+D^{-1} m \\
c^{2}
\end{array}\right](v, Z)=\sum_{\lambda \in \mathbb{Z}^{g}} \hat{f}(\lambda)
$$

Using [7], Ch. II, Lemma 5.8, by substituting $x^{\prime}=x+c^{1}+D^{-1} m$ we get

$$
\hat{f}(\lambda)=e\left(-2 \pi i^{t}\left(c^{1}+D^{-1} m\right) \lambda\right)\left(\operatorname{det} \frac{Z}{i}\right)^{-\frac{1}{2}} e\left(-\pi i^{t}\left(v+c^{2}+\lambda\right) Z^{-1}\left(v+c^{2}+\lambda\right)\right) .
$$

Therefore

$$
\begin{aligned}
& \theta\left[\begin{array}{c}
c^{1}+D^{-1} m \\
c^{2}
\end{array}\right](v, Z)=e\left(-\pi i^{t}\left(v+c^{2}\right) Z^{-1}\left(v+c^{2}\right)\right)\left(\operatorname{det} \frac{Z}{i}\right)^{-\frac{1}{2}} \\
& \cdot \sum_{\lambda \in \mathbb{Z}^{g}} e\left(-2 \pi i^{t}\left(c^{1}+D^{-1} m\right) \lambda-2 \pi i^{t}\left(v+c^{2}\right) Z^{-1} \lambda-\pi i^{t} \lambda Z^{-1} \lambda\right) .
\end{aligned}
$$


By substituting $-\lambda=D k+n, n \in \mathbb{Z}_{D}$, we can rewrite the last sum as

$$
\begin{array}{r}
\sum_{n \in \mathbb{Z}_{D}} \sum_{k \in \mathbb{Z}^{g}} e\left(2 \pi i^{t}\left(c^{1}+D^{-1} m\right)(D k+n)+2 \pi i^{t}\left(v+c^{2}\right) Z^{-1}(D k+n)\right. \\
\left.-\pi i^{t}(D k+n) Z^{-1}(D k+n)\right) .
\end{array}
$$

A straightforward calculation yields

$$
\begin{gathered}
\theta\left[\begin{array}{c}
c^{1}+D^{-1} m \\
c^{2}
\end{array}\right](v, Z)=e\left(-\pi i^{t} v Z^{-1} v\right)\left(\operatorname{det} \frac{Z}{i}\right)^{-\frac{1}{2}} e\left(2 \pi i^{t} c^{1} c^{2}\right) \\
\cdot \sum_{n \in \mathbb{Z}_{D}} e\left(2 \pi i^{t} m D^{-1} n\right) \theta\left[\begin{array}{c}
-D^{-1} c^{2}+D^{-1} n \\
D c^{1}
\end{array}\right]\left(v^{\prime}, Z^{\prime}\right) .
\end{gathered}
$$

Comparing relations (3) and (44), we deduce that the matrix $C(M)$ we are asking for has $m, n$ entry equal to $\left(\frac{d}{i^{g}}\right)^{-\frac{1}{2}} e\left(2 \pi i^{t} m D^{-1} n\right)$. Let $d:=\operatorname{det} D$. The matrix $A=$ $\left(a_{m n}\right)_{m, n \in \mathbb{Z}_{D}}$, where $a_{m n}:=e\left(2 \pi i^{t} m D^{-1} n\right)$, has determinant $\operatorname{det} A=\zeta_{4} d^{\frac{d}{2}}$. To see this, we denote by $\mathbb{C}\left[\mathbb{Z}_{d_{i}}\right]$ the $\mathbb{C}$-vector space of dimension $d_{i}$ "corresponding" to the group $\mathbb{Z}_{d_{i}}$. Fix the natural base $\left\langle m, m \in \mathbb{Z}_{d_{i}}\right\rangle$ and define the map $\phi_{i}: \mathbb{C}\left[\mathbb{Z}_{d_{i}}\right] \longrightarrow$ $\mathbb{C}\left[\mathbb{Z}_{d_{i}}\right]$ by $\phi_{i}(m):=\sum_{n \in \mathbb{Z}_{d_{i}}} e\left(2 \pi i n d_{i}^{-1} m\right) n$. Let $C_{i}$ be the matrix corresponding to $\phi_{i}$. Then $\operatorname{det} C_{i}=\zeta_{4} d_{i}^{d_{i} / 2}$. Indeed, it is easy to see that $\operatorname{det}\left(C_{i}^{2}\right)= \pm d_{i}^{d_{i}}$. Observe now that $A$ is the matrix corresponding to the tensor product of the maps $\phi_{i}$ and so, $\operatorname{det} A=\operatorname{det} C_{1}^{d / d_{1}} \cdots \operatorname{det} C_{g}^{d / d_{g}}=\zeta_{4} d^{d / 2}$. To conclude this case, observe that $\left(\frac{d}{i^{g}}\right)^{-\frac{d}{2}}=\zeta_{8} d^{-\frac{d}{2}}$ and therefore $\operatorname{det} C(M)=\zeta_{8}$.

Matrices in $G_{D}^{\text {int }}$. Let $M \in G_{D}^{\text {int }}$. Then $M$ corresponds to an isomorphism $\psi$ : $X_{Z^{\prime}} \longrightarrow X_{Z}$ which is a lift of an isomorphism of principally polarized abelian varieties. In this case, the usual theta transformation formula holds ([5], Ch. 8, $\S 6)$. Let $a=c+Z w^{1}, c \in \frac{1}{2} \Lambda(H), w^{1}=D^{-1} w_{1} \in D^{-1} \mathbb{Z}^{g}$. We denote by $M[]_{I}$ the transformation of the characteristic corresponding to the principal polarization $D=I$. Note that $M[c]_{I}=M[c]+Z^{\prime} s^{1}$, where $s^{1}:=-\frac{D-I}{2}\left({ }^{t} \gamma \delta\right)_{0}$, and so $s^{1}=$ $D^{-1} s_{1}$, with $s_{1} \in \mathbb{Z}^{g}$. We have the following facts ([5], Ch. $8, \S \S 4$ and 6 ).

1. $\psi^{*} \theta^{a}(v, Z)=C(Z, M, a) \theta^{M[a]_{I}}\left(v^{\prime}, Z^{\prime}\right)$.

2. $C(Z, M, a)=C(Z, M, 0) e\left(\pi i E\left(M[0]_{I}, A^{-1} a\right)\right)$, where $A={ }^{t}(\gamma Z+\delta)$.

3. $C(Z, M, 0)=k(M) e\left(\pi i^{t} M[0]_{I}^{1} M[0]_{I}^{2}\right) \operatorname{det}(\gamma Z+\delta)^{-\frac{1}{2}}$, where $k(M)$ is a $\zeta_{8}$.

Note that $M[a]_{I}^{1}=M[c]_{I}^{1}+\delta w^{1}$ and $M[a]_{I}^{2}=M[c]_{I}^{2}-\beta w^{1}$. The above formulae and the formulae in Section 1 yield that item 1 above becomes

$$
\begin{aligned}
& e\left(-\pi i^{t} c^{2} w^{1}\right) \psi^{*} \theta \frac{c}{Z w^{1}}(v, Z)=C(Z, M, 0) e\left(\pi i E\left(M[0]_{I}, A^{-1} a\right)\right) \\
& \quad \cdot e\left(-\pi i^{t}\left(\delta w^{1}\right) M[c]_{I}^{2}+\pi i^{t} M[c]_{I}^{1}\left(-\beta w^{1}\right)+\pi i^{t}\left(\delta w^{1}\right)\left(-\beta w^{1}\right)\right) \theta \frac{M[c]_{I}}{Z^{\prime} \delta w^{1}}\left(v^{\prime}, Z^{\prime}\right) .
\end{aligned}
$$

Item 5e) of Section 1 gives

$$
\theta \frac{M[c]_{I}}{Z^{\prime} \delta w^{1}}\left(v^{\prime}, Z^{\prime}\right)=e\left(-\pi i^{t} s^{1} M[c]^{2}\right) \theta_{\frac{M[c]}{Z^{\prime}\left(\delta w^{1}+s^{1}\right)}}\left(v^{\prime}, Z^{\prime}\right)
$$


Also,

$$
\begin{gathered}
M[0]_{I}^{1}=\frac{1}{2}\left(\gamma^{t} \delta\right)_{0} \in \frac{1}{2} \mathbb{Z}^{g}, \quad M[0]_{I}^{2}=\frac{1}{2}\left(\alpha^{t} \beta\right)_{0} \in \frac{1}{2} \mathbb{Z}^{g}, \\
\left(A^{-1} a\right)^{1}=\delta\left(c^{1}+w^{1}\right)-\gamma c^{2}, \quad\left(A^{-1} a\right)^{2}=-\beta\left(c^{1}+w^{1}\right)+a c^{2}, \\
M[c]_{I}^{1}=\delta c^{1}-\gamma c^{2}+\frac{1}{2}\left(\gamma^{t} \delta\right)_{0} \in \frac{D^{-1}}{2} \mathbb{Z}^{g}, \\
M[c]_{I}^{2}=-\beta c^{1}+a c^{2}+\frac{1}{2}\left(\alpha^{t} \beta\right)_{0} \in \frac{1}{2} \mathbb{Z}^{g} .
\end{gathered}
$$

We thus get

(5) $\psi^{*} \theta \frac{c}{Z D^{-1} w_{1}}(v, Z)$

$$
=k(M) e(\pi i k)\left(\pi i \lambda w^{1}\right) e\left(-\pi i^{t} w^{1 t} \delta \beta w^{1}\right) \operatorname{det}(\gamma Z+\delta)^{-\frac{1}{2}} \frac{M[c]}{Z^{\prime} D^{-1}\left(\Delta w_{1}+s_{1}\right)}\left(v^{\prime}, Z^{\prime}\right),
$$

where $k={ }^{t} M[0]_{I}^{1} M[0]_{I}^{2}+{ }^{t} M[0]_{I}^{1}\left(-\beta c^{1}+a c^{2}\right)-{ }^{t} M[0]_{I}^{2}\left(\delta c^{1}-\gamma c^{2}\right)-{ }^{t} s^{1} M[c]^{2}$ and $\lambda=-{ }^{t} M[0]_{I}^{1} \beta-{ }^{t} M[0]_{I}^{2} \delta-{ }^{t} M[c]_{I}^{2} \delta-{ }^{t} M[c]_{I}^{1} \beta+{ }^{t} c^{2}$. Observe now that $k \in \frac{1}{4 d_{g}} \mathbb{Z}$ and $\lambda \in \frac{1}{2} \mathbb{Z}$.

Note that when $\gamma \in M_{g}(\mathbb{Z})$, i.e. $\Gamma=D \Gamma_{1}$ for some integral matrix $\Gamma_{1}$, the matrix $\Delta$ acts as a permutation on $\mathbb{Z}_{D}$. Indeed, the relation $\Delta D^{t} A-\Gamma D^{t} B=D$ implies $\Delta\left(D^{t} A D^{-1}\right)=I+\Gamma\left(D^{t} B D^{-1}\right)$ i.e. $\Delta\left(D^{t} A D^{-1}\right)=I+D \Gamma_{1}\left(D^{t} B D^{-1}\right)$ and so $\Delta A_{1}=I+D M$ for some integral matrices $A_{1}, M$. Hence, $\Delta$ induces an epimorphism on $\mathbb{Z}_{D}$ and so an automorphism.

Relation (5) implies that the matrix $C(M)$ of the proposition has in the $w_{1}, \Delta w_{1}+$ $s_{1}$-entry the value $k(M) e(\pi i k) e\left(\pi i \lambda D^{-1} w_{1}\right) e\left(-\pi i^{t} w_{1}{ }^{t} \Delta D^{-1} B w_{1}\right)$ and any other entries in the $w_{1}$ row and $\Delta w_{1}+s_{1}$ column are zero. To find its determinant, we first note that

$$
\prod_{w_{1} \in \mathbb{Z}_{D}} e\left(\pi i \lambda D^{-1} w_{1}\right)=e\left(\pi i \sum_{i=1}^{g} \frac{\lambda_{i}}{d_{i}} \sum_{w_{1} \in \mathbb{Z}_{D}} w_{1, i}\right)=e\left(\pi i \sum_{i=1}^{g} \frac{\lambda_{i}}{d_{i}} \frac{d}{d_{i}} \frac{d_{i}\left(d_{i}-1\right)}{2}\right) .
$$

The above sum belongs to $\frac{1}{4} \mathbb{Z}$, and so the product is a $\zeta_{8}$. Also, the matrix ${ }^{t} \Delta D^{-1} B=D^{-1}\left(D^{t} \Delta D^{-1}\right) B$ is symmetric; let $\alpha_{i j}=\frac{a_{i j}}{d_{i}}, a_{i j} \in \mathbb{Z}$ be its $i j$-entry. Then

$$
\begin{aligned}
& \prod_{w_{1} \in \mathbb{Z}_{D}} e\left(-\pi i^{t} w_{1}{ }^{t} \Delta D^{-1} B w_{1}\right) \\
& \quad=e\left(-\pi i \sum_{i=1}^{g} \frac{a_{i i}}{d_{i}} d \frac{\left(d_{i}-1\right)\left(2 d_{i}-1\right)}{6}-2 \sum_{1 \leq i<j \leq g} \frac{a_{i j}}{d_{i}} d \frac{\left(d_{i}-1\right)\left(d_{j}-1\right)}{4}\right) .
\end{aligned}
$$

The above sums belong to $\frac{1}{2} \mathbb{Z}$, and so the product is a $\zeta_{4}$, except when $3 \mid d_{g}$ and $\left(d_{g-1}, 3\right)=1$, in which case it belongs to $\frac{1}{6} \mathbb{Z}$ and the product is a $\zeta_{12}$. To conclude, we have $\operatorname{det} C(M)=\zeta_{8}$, except when $3 \mid d_{g}$ and $\left(d_{g-1}, 3\right)=1$, in which case $\operatorname{det} C(M)=\zeta_{24}$.

Next, for the case of a totally symmetric bundle, note first that such a bundle always has characteristic in $\Lambda(H)$. Moreover, in Lemma 2.1 if $L\left(H_{Z}, \chi_{0}\right)$ has characteristic in $\Lambda(H)$, then $\psi^{*} L\left(H_{Z}, \chi_{0}\right)$ has also characteristic in $\Lambda(H)$. Indeed, in the case of an "even" polarization, we always have that $\chi_{0}=1$, and so $\psi_{r}^{*} \chi_{0}=$ $1=\chi_{0}^{\prime}$. 
Proposition 2.2. Keeping the notation of Proposition [2.1, we assume in addition that $\mathcal{L}$ is totally symmetric and that $g \geq 3$. Then the matrix $C$, for which $\psi^{*} B^{Z}=$ $C B^{Z^{\prime}}$, is of the form $C=(\operatorname{det}(\gamma Z+\delta))^{-\frac{1}{2}} C(M)$, where $C(M)$ depends on $M$ and $\operatorname{det} C(M)=1$, except when $3 \mid d_{g}$ and $\left(d_{g-1}, 3\right)=1$, in which case we have $\operatorname{det} C(M)=\zeta_{3}$.

Proof. The proof is a modification of the proof of Proposition 2.1:

At the end of the subsection "Matrices of the form $\left(\begin{array}{cc}0 & -D \\ D^{-1} & 0\end{array}\right)$ ": For $g \geq 3$ the number $\frac{d}{d_{i}}$ is a multiple of 4 , and so $\operatorname{det} C_{i}^{\frac{d}{d_{i}}}=d_{i}^{\frac{d}{2}}$. Therefore $\operatorname{det} A=d^{\frac{d}{2}}$. Also, for $g \geq 3$ we have $\left(\frac{d}{i^{g}}\right)^{-\frac{d}{2}}=d^{-\frac{d}{2}}$ Therefore, $\operatorname{det} C(M)=1$.

At the end of the subsection "Matrices in $G_{D}^{\text {int }}$ ": The sum in relation (6) is an even integer, and so the product is 1 . For $g \geq 3$, the right summand in relation (77) is an even integer. The left summand is an even integer, except when $3 \mid d_{g}$ and $\left(d_{g-1}, 3\right)=1$, in which case it belongs to $\frac{2}{3} \mathbb{Z}$. Therefore the product is 1 , except when $3 \mid d_{g}$ and $\left(d_{g-1}, 3\right)=1$, in which case it is $\zeta_{3}$. Also $k(M)^{d}=1$. Thus, to show that $\operatorname{det} C(M)=1$ (resp. $\zeta_{3}$ ), it suffices to show that the permutation of $\mathbb{Z}_{D}$ induced by the action of $\Delta$ followed by the addition by the vector $s_{1}$ has sign 1 .

We show first that $\operatorname{sgn}(\Delta)=1$. Indeed, let $d_{i}=2^{k_{i}} m_{i}$, with $1 \leq k_{1} \leq k_{2} \leq \cdots \leq$ $k_{g}$ and $m_{1}\left|m_{2}\right| \cdots \mid m_{g}$ odd integers. Define $\mathbb{Z}_{\mathrm{ev}}:=\mathbb{Z}_{2^{k_{1}}} \oplus \cdots \oplus \mathbb{Z}_{2^{k_{g}}}$, a group of order $n_{\text {ev }}$, and $\mathbb{Z}_{\text {odd }}:=\mathbb{Z}_{m_{1}} \oplus \cdots \oplus \mathbb{Z}_{m_{g}}$, a group of order $n_{\text {odd }}$. Then $\mathbb{Z}_{D}=\mathbb{Z}_{\text {ev }} \oplus \mathbb{Z}_{\text {odd }}$. Let $\phi: \mathbb{Z}_{D} \longrightarrow \mathbb{Z}_{D}$ be an automorphism. Then $\phi\left(\mathbb{Z}_{\text {ev }}\right)=\mathbb{Z}_{\text {ev }}$ and $\phi\left(\mathbb{Z}_{\text {odd }}\right)=\mathbb{Z}_{\text {odd }}$. We denote by $\phi_{\mathrm{ev}}$ (resp. $\phi_{\text {odd }}$ ) the restriction of $\phi$ to $\mathbb{Z}_{\mathrm{ev}}$ (resp. to $\mathbb{Z}_{\text {odd }}$ ). If we interpret $\phi$ as a linear automorphism of $\mathbb{C}\left[\mathbb{Z}_{D}\right]$, then $\phi=\phi_{\mathrm{ev}} \otimes \phi_{\text {odd }}$, and so $\operatorname{sgn} \phi=\operatorname{sgn} \phi_{\mathrm{ev}}^{n_{\text {odd }}} \operatorname{sgn} \phi_{\mathrm{odd}}^{n_{\mathrm{ev}}}$. But $n_{e v}$ is an even number; hence it suffices to prove the result for $\mathbb{Z}_{D}=\mathbb{Z}_{\mathrm{ev}}$.

Let $E$ be the matrix which corresponds to the automorphism $\phi_{\mathrm{ev}}$. We call elementary transformations of $\mathbb{Z}_{\mathrm{ev}}$ those which correspond to left or right multiplication by a matrix of one of the following types: 1 in the diagonal and $a_{i j} \in \mathbb{Z}$ in some $i j$-entry with $j \geq i$; or 1 in the diagonal and $2^{k_{i}-k_{j}} a_{i j}, a_{i j} \in \mathbb{Z}$, in some $i j$-entry with $j<i$ (and zero everywhere else). We then claim that by multiplying the matrix $E=\left(e_{i j}\right)$ with the above type of matrices, we can produce a matrix with all the elements of the last row, except the diagonal one, equal to zero $\bmod 2^{k_{g}}$ and the $i$-th element of the last column, with $1 \leq i<g$, zero $\bmod 2^{k_{i}}$. Indeed, we may first assume that $e_{g g}$ is an odd integer: the determinant of $E$ is an odd number since $E$ defines an automorphism, and so some of the elements of the last row must be odd. If $e_{g g}$ is even, let $e_{g j_{0}}, j_{0}<g$, be the odd element. But then, using an elementary transformation, we can add the $j_{0}$-th column to the last one, and so the $g g$-entry becomes odd. Since $D^{-1} E D$ is an integral matrix, we have that $e_{g j}=2^{k_{g}-k_{j}} m_{g j}, m_{g j} \in \mathbb{Z}$. But now the equation $2^{k_{g}-k_{j}} e_{g g} x \equiv-2^{k_{g}-k_{j}} m_{g j} \bmod 2^{k_{g}}$ has a solution, and therefore by multipling the matrix $E$ on the right by the elementary matrix which has $2^{k_{g}-k_{j}} x$ in the $g j$-entry, we get that the $g j$-entry of the product is zero $\bmod 2^{k_{g}}$. On the other hand, by multiplying on the left by an elementary matrix which has $x$ in the $i g$-entry, where $x$ is the solution of $c_{g g} x \equiv-c_{i g} \bmod 2^{k_{i}}$, we get that the $i g$-entry of the product is zero $\bmod 2^{k_{i}}$.

A matrix like the one we produced corresponds to an even permutation of $\mathbb{Z}_{\mathrm{ev}}$. Indeed, by writing $\mathbb{Z}_{\mathrm{ev}}$ as a direct sum of two groups, the second of which is the $\mathbb{Z}_{2^{k_{g}}}$, 
we get the action is a direct sum of actions. We thus get that the signature of the permutation is one, since both groups are of even order. A similar argument yields that the action is given by the elementary matrices induces an even permutation (here we have to use the hypothesis $g \geq 3$ ). We therefore get that the permutation given by $\phi_{\text {ev }}$ is an even one.

Finally, the matrix of the permutation of $\mathbb{Z}_{D}$ induced by addition of the vector $s_{1}$ is the tensor product of the matrices corresponding to the permutation of $\mathbb{Z}_{d_{i}}$ induced by addition of $s_{i}^{1}$. Since the size of all those matrices is an even number, the determinant of the tensor product is 1 . This concludes the proof.

\section{Abelian fibrations}

Everything we have stated which holds for a fixed abelian variety $X=V / \Lambda$ can be transferred easily over a fibration $X \longrightarrow U$ of abelian varieties of type $D$, with base $U$ a simply connected Stein manifold (such as $\mathfrak{h}_{g}$ ). In this case, the universal covering $\tilde{X}$ of $X$ will take the place of $V$ and the homotopy group $\pi_{1}(X)$ the place of $\Lambda$. When the base is the space $\mathfrak{h}_{g}$, there exists a universal family $\mathfrak{X} \longrightarrow \mathfrak{h}_{g}$, with fiber over $Z$ the abelian variety $X_{Z}$. It is defined as the quotient of $\mathbb{C}^{g} \times \mathfrak{h}_{g}$ by the action of $\Lambda_{D}=\mathbb{Z}^{g} \oplus D \mathbb{Z}^{g}$ given by $l(v, Z)=\left(v+j_{Z}(l), Z\right)$. We have $\pi_{1}\left(\mathfrak{X}_{D}\right)=\Lambda_{D}$ and $\tilde{\mathfrak{X}}_{D}=\mathbb{C}^{g} \times \mathfrak{h}_{g}$. Suppose $\left(c^{1}, c^{2}\right) \in \mathbb{R}^{g} \oplus \mathbb{R}^{g}$ and let $c(Z)=Z c^{1}+c^{2}$. For each such $c=c(Z)$, we have on $\mathfrak{X}_{D}$ a line bundle $\mathcal{L}_{\mathfrak{X}}^{c}$ corresponding to the classical factor of automorphy $e_{c}: \Lambda_{D} \times\left(\mathbb{C}^{g} \times \mathfrak{h}_{g}\right) \longrightarrow \mathbb{C}^{\times}$of characteristic $c$, given by $e_{c}(l ; v, Z)=e\left(2 \pi i\left({ }^{t} c^{1} \lambda^{2}-{ }^{t} c^{2} \lambda^{1}\right)-\pi i^{t} \lambda^{1} Z \lambda^{1}-2 \pi i^{t} v \lambda^{1}\right)$, where $l=\left(\lambda^{1}, \lambda^{2}\right) \in \Lambda_{D}=\mathbb{Z}^{g} \oplus D \mathbb{Z}^{g}$. Note that $e_{c}$, as well as $\mathcal{L}_{\mathfrak{X}}^{c}$, depends only on the class $\left(c^{1}, c^{2}\right) \bmod \left(D^{-1} \mathbb{Z}^{g} \oplus \mathbb{Z}^{g}\right)$.

3.1. Line bundles on abelian fibrations. Let $\mathrm{f}: X \longrightarrow B$ be a fibration of abelian varieties and $\mathcal{L}$ a symmetric line bundle on $X$, trivialized along the zero section, which defines a polarization of type $D$ on each fiber. We denote by $s$ : $B \longrightarrow X$ the zero section and let $S:=s(B)$.

We denote by $\tilde{B}$ the universal covering of $B$. There exist a period map $p: \tilde{B} \longrightarrow$ $\mathfrak{h}_{g}$ and a representation $\rho: \pi_{1}(B) \longrightarrow G_{D}$ of $B$. The choice of $p$ and $\rho$ is unique, up to the action by a fixed element of $G_{D}$. Let $Y:=\mathfrak{X}_{D} \times_{\mathfrak{h}_{g}} \tilde{B}$, and $\tilde{\mathrm{f}}: Y \longrightarrow \tilde{B}$ the induced map. There is a canonical map $\pi_{1}: Y \longrightarrow X$ which makes the following diagram commutative:

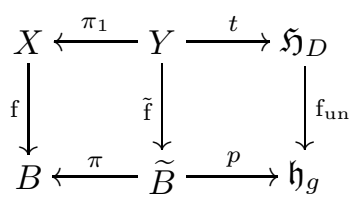

For each $\tilde{b} \in \tilde{B}$ and $\sigma \in \pi_{1}(B)$, the period map $p$ satisfies $p(\sigma \tilde{b})=\rho(\sigma) \cdot p(\tilde{b})$, where - denotes the action of $G_{D}$ on $\mathfrak{h}_{g}$. We use $Z(\tilde{b})$ to denote the matrix $p(\tilde{b})$. If $M=\rho(\sigma)$, then the above relation translates to $Z(\sigma \tilde{b})=M(Z(\tilde{b}))$, as defined in Section 1

The group $\Lambda_{D}$ acts on $\tilde{B} \times \mathbb{C}^{g}$ by $l(\tilde{b}, v)=\left(\tilde{b}, v+j_{Z(\tilde{b})}(l)\right)$. The quotient of $\tilde{B} \times \mathbb{C}^{g}$ by this action, the elements of which we denote by $[\tilde{b}, v]$, is naturally isomorphic to $Y$, and the canonical map $\tilde{B} \times \mathbb{C}^{g} / \Lambda_{D} \longrightarrow \tilde{B}$ is identified with $\tilde{\mathrm{f}}$. The group $\pi_{1}(B)$ acts on $Y$ by $\sigma[\tilde{b}, v]=\left[\sigma \tilde{b}, M_{Z(\tilde{b})}(v)\right]$, where $M=\rho(\sigma)$. The action is free and 
properly discontinuous, the quotient is isomorphic to $X$, and the canonical map $Y \longrightarrow Y / \pi_{1}(B)$ is identified with $\pi_{1}$. The above action defines an isomorphism $\phi_{\sigma}: Y_{\tilde{b}} \longrightarrow Y_{\sigma \tilde{b}}$. When we identify $Y_{\tilde{b}}$ with $X_{Z(\tilde{b})}$ and $Y_{\sigma \tilde{b}}$ with $X_{M(Z(\tilde{b}))}$, the above map becomes the map $\phi(M)$.

Let $\pi_{1}^{*} \mathcal{L}$ be the pull back of the line bundle $\mathcal{L}$ to $Y$. A symmetric line bundle $L(H, \chi)$ always has characteristic $c \in \frac{1}{2} \Lambda(H)$ w.r.t. any decomposition of $H$, since $\chi(\lambda)= \pm 1$ for all $\lambda \in \Lambda$. Therefore, a characteristic of the restriction of $\pi_{1}^{*} \mathcal{L}$ to the fiber over $\tilde{b}$, w.r.t. the decomposition induced by the period map $p$, is of type $c=Z(\tilde{b}) c^{1}+c^{2}$, where $\left(c^{1}, c^{2}\right) \in \frac{1}{2}\left(D^{-1} \mathbb{Z}^{g} \oplus \mathbb{Z}^{g}\right)$, and, by continuity, the class $\left(c^{1}, c^{2}\right) \bmod \left(D^{-1} \mathbb{Z}^{g} \oplus \mathbb{Z}^{g}\right)$ is independent of the choice of $\tilde{b}$. Note that when $\mathcal{L}_{X}$ is totally symmetric, then $\left(c^{1}, c^{2}\right) \in D^{-1} \mathbb{Z}^{g} \oplus \mathbb{Z}^{g}$. For each $\sigma \in \pi_{1}(B)$, the pull back of the isomorphism $\phi_{\sigma}$ defines an isomorphism of the total space of $\pi_{1}^{*} \mathcal{L}$. If $M=\rho(\sigma)$, then $\phi_{\sigma}=\phi(M)$, and so, $M$ "preserves" the characteristic, i.e. $\left(M[c]^{1}, M[c]^{2}\right)=\left(c^{1}, c^{2}\right) \bmod \left(D^{-1} \mathbb{Z}^{g} \oplus \mathbb{Z}^{g}\right)$.

The group $\Lambda_{D}$ acts on $\tilde{B} \times \mathbb{C}^{g} \times \mathbb{C}$ by $l(\tilde{b}, v, z)=\left(\tilde{b}, v+j_{Z(\tilde{b})}(l), e_{c}(l ; v, Z(\tilde{b})) z\right)$. The quotient $\tilde{\mathcal{L}}^{c}$ is a line bundle on $Y$, the elements of which we denote by $[\tilde{b}, v, z]$. By construction, $\tilde{\mathcal{L}}^{c}=t^{*} \mathcal{L}_{\mathfrak{X}}^{c}$. The group $\pi_{1}(B)$ acts on $\tilde{\mathcal{L}}^{c}$ by $\sigma[\tilde{b}, v, z]=$ $\left[\sigma \tilde{b}, M_{Z(\tilde{b})}(v), h(v)^{-1} z\right]$, where $M=\rho(\sigma)$ and $h$ is the function introduced in Lemma 2.1, and its value is taken w.r.t. the element $Z(\tilde{b}) \in \mathfrak{h}_{g}$ and the matrix $M$. To see that the action is well defined, one has to use Lemma 2.1 combined with the fact that the action of $\pi_{1}(B)$ "preserves" the characteristic.

Let $\phi_{\sigma}: Y_{\tilde{b}} \longrightarrow Y_{\sigma \tilde{b}}$ be the map defined above. We fix the identification $\left(\pi_{1}\left(Y_{\tilde{b}}\right), \tilde{Y}_{\tilde{b}}\right) \cong\left(\Lambda_{D}, \mathbb{C}^{g}\right)$ via the map $p$. Then, the line bundle $\left.\tilde{\mathcal{L}}^{c}\right|_{Y_{\tilde{b}}}$ corresponds to the factor of automorphy $e_{c}$ and the line bundle $\phi_{\sigma}^{*}\left(\left.\tilde{\mathcal{L}}^{c}\right|_{Y_{\tilde{b}}}\right)$ corresponds to $\phi_{\sigma}^{*} e_{c}$. From Lemma 2.1 we have that $\phi_{\sigma}^{*} e_{c}=e_{c} \star h$. The action of $\sigma$ on $\tilde{\mathcal{L}}^{c}$ then induces an isomorphism $\Phi_{\sigma}:\left.\tilde{\mathcal{L}}^{c}\right|_{Y_{\tilde{b}}} \longrightarrow \phi_{\sigma}^{*}\left(\left.\tilde{\mathcal{L}}^{c}\right|_{Y_{\sigma \tilde{b}}}\right)$, which is the canonical isomorphism $\Phi_{h}$ defined in Section 1

We claim that the quotient of the line bundle $\tilde{\mathcal{L}}^{c}$ by the above action of $\pi_{1}(B)$ is a line bundle $\mathcal{L}^{c}$ on $X$ isomorphic to $\mathcal{L}$. This is a consequence of the see-saw principle. Indeed, the restrictions of $\mathcal{L}$ and $\mathcal{L}^{c}$ to the fibers of $\mathrm{f}$ are isomorphic, since they have the same characteristic. Also, by definition, $\mathcal{L}$ is trivial on the zero section $S$; the same holds for $\mathcal{L}^{c}$ since, if $\tilde{S}$ is the lift of $S$ on $Y$, then the restricted action of $\pi_{1}(B)$ on $\tilde{S}$ is given by $\sigma[\tilde{b}, 0, z]=[\sigma \tilde{b}, 0, z]$ and therefore the quotient is the trivial bundle. In the following, we identify $\mathcal{L}$ with $\mathcal{L}^{c}$. Finally, the action of $\sigma$ defines an isomorphism $\Psi_{\sigma}$ of $H^{0}\left(Y_{\tilde{b}},\left.\tilde{\mathcal{L}}^{c}\right|_{Y_{\tilde{b}}}\right)$ with $H^{0}\left(Y_{\sigma \tilde{b}},\left.\tilde{\mathcal{L}}^{c}\right|_{Y_{\sigma \tilde{b}}}\right)$, which is induced by the map $\Phi_{\sigma}^{*} \phi_{\sigma}^{*}$. Next we determine the matrix $\tilde{C}^{\sigma}(\tilde{b})$ of $\Psi_{\sigma}$ in terms of given bases.

The functions $\theta\left[\begin{array}{c}c^{1}+D^{-1} m \\ c^{2}\end{array}\right](v, Z(\tilde{b})), m \in \mathbb{Z}_{D}$, are theta functions for the classical factor of automorphy $e_{c}: \Lambda_{D} \times\left(\mathbb{C}^{g} \times p(\tilde{B})\right) \longrightarrow \mathbb{C}^{\times}$, and the line bundle $\tilde{\mathcal{L}}^{c}$ corresponds, by construction, to $e_{c}$. Let $\tilde{s}_{m}$ denote the section of $\tilde{\mathcal{L}}^{c}$ corresponding to the above theta function. The set $\mathcal{B}^{\tilde{b}}:=\left\langle\tilde{s}_{m}^{\tilde{b}}:=\left.\tilde{s}_{m}\right|_{Y_{\tilde{b}}}, m \in \mathbb{Z}_{D}\right\rangle$ forms a base of sections of $H^{0}\left(Y_{\tilde{b}},\left.\tilde{\mathcal{L}}^{c}\right|_{Y_{\tilde{b}}}\right)$ for every $\tilde{b} \in \tilde{B}$. Let $\mathcal{B}^{\sigma \tilde{b}}:=\left\langle\tilde{s}_{n}^{\sigma \tilde{b}}:=\left.\tilde{s}_{n}\right|_{Y_{\sigma \tilde{b}}}, n \in \mathbb{Z}_{D}\right\rangle$ be the corresponding base for $H^{0}\left(Y_{\sigma \tilde{b}},\left.\tilde{\mathcal{L}}^{c}\right|_{Y_{\sigma \tilde{b}}}\right)$. Let $\mathcal{B}_{1}^{\tilde{b}}:=\left\langle\Phi_{\sigma}^{*} \phi_{\sigma}^{*} \tilde{s}_{n}^{\sigma \tilde{b}}, n \in \mathbb{Z}_{D}\right\rangle$; this is also a base for $H^{0}\left(Y_{\tilde{b}},\left.\tilde{\mathcal{L}}^{c}\right|_{\tilde{b}}\right)$. Then the matrix $\tilde{C}^{\sigma}(\tilde{b})$ of $\Psi_{\sigma}$ in the above bases satisfies the relation $\mathcal{B}_{1}^{\tilde{b}}=\tilde{C}^{\sigma}(\tilde{b}) \mathcal{B}^{\tilde{b}}$. 
THETA LINE BUNDLES AND THE DETERMINANT OF THE HODGE BUNDLE 2565

Let $Z:=Z(\tilde{b}), Z^{\prime}:=M(Z(\tilde{b}))$ and $v^{\prime}:=M_{Z(\tilde{b})}(v)$. The section $\Phi_{\sigma}^{*} \phi_{\sigma}^{*} \tilde{s}_{m}^{\sigma \tilde{b}}$ corresponds to the theta function $h(v) \theta\left[\begin{array}{c}c^{1}+D^{-1} n \\ c^{2}\end{array}\right]\left(v^{\prime}, Z^{\prime}\right)$. The above relation of bases becomes

$$
\begin{aligned}
h(v) & \left\langle\theta\left[\begin{array}{c}
c^{1}+D^{-1} n \\
c^{2}
\end{array}\right]\left(v^{\prime}, Z^{\prime}\right), n \in \mathbb{Z}_{D}\right\rangle \\
& =\tilde{C}^{\sigma}(\tilde{b})\left\langle\theta\left[\begin{array}{c}
c^{1}+D^{-1} m \\
c^{2}
\end{array}\right](v, Z), m \in \mathbb{Z}_{D}\right\rangle .
\end{aligned}
$$

We write the matrix $M$ in the form $M=\left(\begin{array}{cc}\alpha & \beta \\ \gamma & \delta\end{array}\right)$. Let $c^{1}:=M[c]^{1}+s^{1}$ and $c^{2}:=M[c]^{2}+s^{2}$, where $s^{1}=D^{-1} s_{1}$ and $s^{2}=s_{2}$, with $s_{1}, s_{2} \in \mathbb{Z}^{g}$. Then item 5b) in Section 1 yields

$$
\begin{gathered}
\theta\left[\begin{array}{c}
c^{1}+D^{-1} n \\
c^{2}
\end{array}\right]\left(v^{\prime}, Z^{\prime}\right)=\theta\left[\begin{array}{c}
M[c]^{1}+D^{-1}\left(n+s_{1}\right) \\
M[c]^{2}+s_{2}
\end{array}\right]\left(v^{\prime}, Z^{\prime}\right) \\
=e\left(2 \pi i^{t} s^{2}\left(M[c]^{1}+D^{-1}\left(n+s_{1}\right)\right) \theta\left[\begin{array}{c}
M[c]^{1}+D^{-1}\left(n+s_{1}\right) \\
M[c]^{2}
\end{array}\right]\left(v^{\prime}, Z^{\prime}\right) .\right.
\end{gathered}
$$

Using relation (2), we now get

$$
\begin{aligned}
\text { (10) } h(v) \theta\left[\begin{array}{c}
c^{1}+D^{-1} n \\
c^{2}
\end{array}\right]\left(v^{\prime}, Z^{\prime}\right) \\
=e\left(2 \pi i^{t} s^{2}\left(M[c]^{1}+s^{1}\right)+\pi i^{t} M[c]^{1} M[c]^{2}-\pi i^{t} c^{1} c^{2}\right)(\operatorname{det}(\gamma Z+\delta))^{\frac{1}{2}} \\
\quad \cdot \sum_{m \in \mathbb{Z}_{D}} e\left(2 \pi i^{t} s^{2} D^{-1} n\right) C(M)_{s_{1}+n, m}^{-1} \theta\left[\begin{array}{c}
c^{1}+D^{-1} m \\
c^{2}
\end{array}\right](v, Z) .
\end{aligned}
$$

The number inside the first exponential is of the form $2 \pi i k$, where $k \in \frac{1}{4 d_{g}} \mathbb{Z}$. A similar calculation as in relation (6) yields that the matrix $A$, with $A_{n m}:=$ $e\left(2 \pi i^{t} s^{2} D^{-1} n\right) C(M)_{s_{1}+n, m}^{-1}$, has determinant $\operatorname{det} A=\zeta_{2} \operatorname{det} C(M)^{-1}$. Comparing relations (9), (10) and using Proposition 2.1] we conclude that $\operatorname{det} \tilde{C}^{\sigma}(\tilde{b})=$ $\zeta_{8}(\operatorname{det}(\gamma Z+\delta))^{\frac{d}{2}}$, except when $3 \mid d_{g}$ and $\operatorname{gcd}\left(3, d_{g-1}\right)=1$, in which case we have $\operatorname{det} \tilde{C}^{\sigma}(\tilde{b})=\zeta_{24}\left(\operatorname{det}(\gamma Z+\delta)^{\frac{d}{2}}\right.$.

In the totally symmetric case we have $\prod_{n \in \mathbb{Z}_{D}^{g}} e\left(2 \pi i^{t} s^{2} D^{-1} n\right)=1$, and the sign of the permutation of $\mathbb{Z}_{D}$ induced by the action "addition of $s_{1}$ " is 1 . Hence, $\operatorname{det} A=$ $\operatorname{det} C(M)^{-1}=1$. We therefore get that $\operatorname{det} \tilde{C}^{\sigma}(\tilde{b})=(\operatorname{det}(\gamma Z+\delta))^{\frac{d}{2}}$, except when $3 \mid d_{g}$ and $\operatorname{gcd}\left(3, d_{g-1}\right)=1$, in which case we have $\operatorname{det} \tilde{C}^{\sigma}(\tilde{b})=\zeta_{3}(\operatorname{det}(\gamma Z+\delta))^{\frac{d}{2}}$.

3.2. Proof of Theorems $\mathbf{A}$ and B. We cover $B$ by small open analytic sets $U^{a}$. We choose $W^{a}$ to be a lift of $U^{a}$ on $\tilde{B}$. Let $\pi_{a}: W^{a} \longrightarrow U^{a}$ be the natural isomorphism. For a point $s \in U^{a}$, we denote by $w^{a}(s)$ its preimage in $W^{a}$. For $s \in U^{a}$, we define $Z^{a}(s):=Z\left(w^{a}(s)\right)$. Let $\left\langle U^{a}, \lambda_{1}^{a}(s), \ldots, \lambda_{2 g}^{a}(s)\right\rangle$ be the choice of a symplectic base on the fibers of $X^{a}:=\mathrm{f}^{-1}\left(U^{a}\right)$, induced by the restriction of the period map $p$ on $W^{a}$. For each $a, b$ with $U^{a b}:=U^{a} \cap U^{b} \neq \emptyset$, there is a matrix $M^{a b}=\left(\begin{array}{cc}\alpha^{a b} & \beta^{a b} \\ \gamma^{a b} & \delta^{a b}\end{array}\right) \in G_{D}$ relating the two symplectic bases. This matrix has the following interpretation. Given $s \in U^{a b}$, there exists a unique $\sigma_{a b} \in \pi_{1}(B)$ such that $\sigma_{a b} w^{a}(s)=w^{b}(s)$ and $M^{a b}=\rho\left(\sigma_{a b}\right)$. Let $\tilde{C}^{a b}(\tilde{b})$ be the matrix $\tilde{C}^{\sigma_{a b}}(\tilde{b})$ defined in Section 3.1 above. The vector bundle $\mathrm{f}_{*} \mathcal{L}$ then has transition matrices 
$g_{a b}^{\mathrm{v}}: U^{a b} \longrightarrow G L(d)$, where $d=\operatorname{det} D$, defined by $g_{a b}^{\mathrm{v}}(s):=\tilde{C}^{a b}\left(w^{a}(s)\right)$. We have thus proven:

Lemma 3.1. Let $\mathrm{f}: X \longrightarrow B$ be a fibration of abelian varieties of relative dimension $g$. Suppose $\mathcal{L}$ is the symmetric (resp. totally symmetric and $g \geq 3$ ) line bundle on $X$, and $\left\{U^{a}\right\}$ is the trivialization of $B$ given above. Then, the transition functions of the line bundle $\operatorname{det} \mathrm{f}_{*} \mathcal{L}$ are given by $g_{\mathcal{L}}^{a b}(s)=\zeta_{8}\left(\operatorname{det}\left(\gamma^{a b} Z^{a}(s)+\delta^{a b}\right)\right)^{\frac{d}{2}}$ (resp. $\left.g_{\mathcal{L}}^{a b}(s)=\left(\operatorname{det}\left(\gamma^{a b} Z^{a}(s)+\delta^{a b}\right)\right)^{\frac{d}{2}}\right)$, except when $3 \mid d_{g}$ and $\operatorname{gcd}\left(3, d_{g-1}\right)=1$, in which case we have that $g_{\mathcal{L}}^{a b}(s)=\zeta_{24}\left(\operatorname{det}\left(\gamma^{a b} Z^{a}(s)+\delta^{a b}\right)\right)^{\frac{d}{2}}\left(\right.$ resp. $g_{\mathcal{L}}^{a b}(s)=$ $\left.\zeta_{3}\left(\operatorname{det}\left(\gamma^{a b} Z^{a}(s)+\delta^{a b}\right)\right)^{\frac{d}{2}}\right)$.

The proof of Theorems $\mathrm{A}$ and $\mathrm{B}$ is now a consequence of the above Lemma 3.1 and the following Lemma 3.2

Lemma 3.2. Let $\mathrm{f}: X \longrightarrow B$ be a fibration of abelian varieties and $s: B \longrightarrow X$ the zero section. Let $\Omega_{X / B}$ denote the relative cotangent bundle. Then $\Omega_{X / B} \cong \mathrm{f}^{*} E$, where $E \cong s^{*} \Omega_{X / B}$ is the vector bundle on $B$ defined by the transition matrices $g_{E}^{a b}:=\left(\gamma^{a b} Z^{a}(s)+\delta^{a b}\right)^{-1}$. In particular, for the relative dualizing sheaf of $\mathrm{f}$ we have that $\omega_{X / B} \cong \mathrm{f}^{*} \mu$, where $\mu \cong s^{*} \omega_{X / B}$ is the line bundle on $B$ defined by the transition functions $g_{\mu}^{a b}(s)=\operatorname{det}\left(\gamma^{a b} Z^{a}(s)+\delta^{a b}\right)^{-1}$.

Proof. The period matrix $Z^{a}(s)$ of $X_{s}^{a}$ satisfies

$$
\left\langle\lambda_{1}^{a}(s), \ldots, \lambda_{g}^{a}(s)\right\rangle=\left\langle\lambda_{g+1}^{a}(s), \ldots, \lambda_{2 g}^{a}(s)\right\rangle Z^{a}(s) .
$$

$\Lambda_{D}$ acts on $\mathbb{C}^{g} \times U^{a}$ by $l(v, s):=\left(v+j_{Z^{a}(s)}(l), s\right)$, where $j_{Z^{a}(s)}(l):=Z^{a}(s) \lambda^{1}+\lambda^{2}$ and $l=\left(\lambda^{1}, \lambda^{2}\right)$. There is a canonical isomorphism $\phi_{a}: X^{a} \longrightarrow\left(\mathbb{C}^{g} \times U^{a}\right) / \Lambda_{D}$ (fibered over $\left.U^{a}\right)$ defined on the level of universal coverings by $\tilde{\phi}_{a}\left(\lambda_{g+i}^{a}(s)\right)=\left(e_{i}, s\right) \in$ $\mathbb{C}^{g} \times U^{a}, i=1, \ldots, g$, where $\left\langle e_{1}, \ldots, e_{g}\right\rangle$ is the standard base of $\mathbb{C}^{g}$. Let $\left\langle z_{1}, \ldots, z_{g}\right\rangle$ denote the standard coordinates of $\mathbb{C}^{g}$. Then $d z_{i}$ is the dual to $e_{i}$. Let $z_{i}^{a}:=$ $\tilde{\phi}_{a}^{*}\left(z_{i} \times i d\right)$. Then $\left\langle d z_{1}^{a}, \ldots, d z_{g}^{a}\right\rangle$ defines at each point of $X^{a}$ a base of sections of the fiber of $\left.\Omega_{X / B}\right|_{X^{a}}$, and $d z_{1}^{a} \wedge \ldots \wedge d z_{g}^{a}$ defines a (nowhere zero) section of $\left.\omega_{X / B}\right|_{X^{a}}$. This is because $d z_{1} \wedge \ldots \wedge d z_{g}$ defines a (nowhere zero) section of the relative dualizing sheaf of the fibration $\left(\mathbb{C}^{g} \times U^{a}\right) / \Lambda_{D} \longrightarrow U^{a}$.

We have that $\left\langle\lambda_{1}^{b}, \ldots, \lambda_{2 g}^{b}\right\rangle=\left\langle\lambda_{1}^{a}, \ldots, \lambda_{2 g}^{a}\right\rangle^{t} M^{a b}$. Therefore

$$
\left\langle\lambda_{g+1}^{b}, \ldots, \lambda_{2 g}^{b}\right\rangle=\left\langle\lambda_{g+1}^{a}, \ldots, \lambda_{2 g}^{a}\right\rangle^{t}\left(\gamma^{a b} Z^{a}(s)+\delta^{a b}\right) .
$$

By taking dual bases and applying determinants we get that

$$
d z_{1}^{b} \wedge \ldots \wedge d z_{g}^{b}=\operatorname{det}\left(\gamma^{a b} Z^{a}(s)+\delta^{a b}\right)^{-1} d z_{1}^{a} \wedge \ldots \wedge d z_{g}^{a}
$$

\section{The JACOBIAN FIBRATION}

We now apply the above considerations to the Jacobian fibration $\mathrm{f}: \mathcal{J} \longrightarrow \mathcal{M}_{g}$, where $\mathcal{J}$ denotes the universal Jacobian parametrizing line bundles of degree zero on the fibers of the universal curve $\psi: \mathcal{C} \longrightarrow \mathcal{M}_{g}$. The Picard group of $\mathcal{M}_{g}$ is freely generated over the integers by the line bundle $\lambda:=\operatorname{det} \psi_{*} \omega_{\mathcal{C} / \mathcal{M}_{g}}$ [1]. Due to the description of the Jacobian of a curve $C$ as $J^{0}(C) \cong \frac{H^{0}\left(C, \omega_{C}\right)^{\vee}}{H_{1}(C, \mathbb{Z})}$, one deduces that $\lambda$ has the same transition functions as $s^{*} \omega_{\mathcal{J} / \mathcal{M}_{g}}$; see e.g. [2], Ch. III, Prop. 17.1, where a slightly different notation is used. Hence $\lambda \cong s^{*} \omega_{\mathcal{J} / \mathcal{M}_{g}}$. 
On the Jacobian fibration $\mathcal{J}$, there is a totally symmetric line bundle $\mathcal{L}$ which restricts to a line bundle of class $2 \theta$ on the fibers and is trivial along the zero section. It is defined as the pull back of the Poincaré bundle under the natural map. Theorem B yields that

Corollary 4.1. With the above notation,

$$
\operatorname{det} \mathrm{f}_{*}\left(\mathcal{L}^{\otimes n}\right) \cong-\frac{(2 n)^{g}}{2} \operatorname{det} \psi_{*} \omega_{\mathcal{C} / \mathcal{M}_{g}}
$$

Remark 4.1. One can also prove Corollary 4.1 by using Theorem 5.1 in 4 ]. The torsion factor in that theorem can be canceled, due to the above mentioned fact about the generator of the Picard group of $\mathcal{M}_{g}$.

4.1. Proof of Theorem C. Let $\mathrm{f}_{g-1}: \mathcal{J}^{g-1} \longrightarrow \mathcal{M}_{g}$ be the Jacobian fibration of degree $g-1$. We use the following result from [8], 9]. Let $\alpha: \tilde{\mathcal{M}}_{g} \longrightarrow \mathcal{M}_{g}$ denote the covering of even theta characteristics in $\mathcal{J}^{g-1}$. It is a covering of degree $2^{g-1}\left(2^{g}+1\right)$. The theta divisor $\Theta$ in $\mathcal{J}^{g-1}$ intersects $\tilde{\mathcal{M}}_{g}$ transversely, and the (set theoretic) intersection projects birationally, via $\alpha$, to a divisor in $\mathcal{M}_{g}$ which has class $2^{g-3}\left(2^{g}+1\right) c_{1}(\lambda)$. On the other hand, the generic point of the intersection corresponds to a line bundle with two sections. By the description of the singularities of the theta divisor, we have that, on such a point, the theta divisor has a singularity of multiplicity 2 . Therefore the push-forward, by $\alpha$, of the (scheme theoretic) intersection of $\Theta$ with $\tilde{\mathcal{M}}_{g}$ is a divisor of class $2^{g-2}\left(2^{g}+1\right) c_{1}(\lambda)$. We use the following commutative diagram:

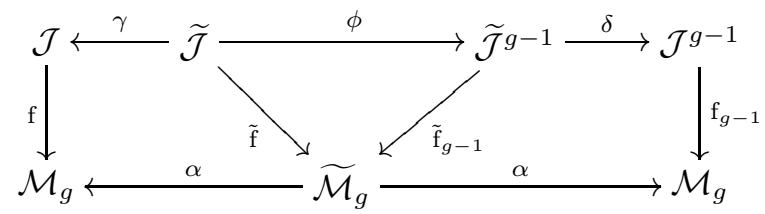

In the diagram we denote by $\tilde{\mathcal{J}}$ and $\tilde{\mathcal{J}}^{g-1}$ the pull back of $\mathcal{J}$ and $\mathcal{J}^{g-1}$ on $\tilde{\mathcal{M}}_{g}$. By $\phi$ we denote the etale map of degree $2^{2 g}$ which sends $L \in \tilde{\mathcal{J}}$, sitting over the point $[C, \eta] \in \tilde{\mathcal{M}}_{g}$, to $L^{\otimes 2} \otimes \eta \in \tilde{\mathcal{J}}^{g-1}$. Let $\tilde{s}: \tilde{\mathcal{M}}_{g} \longrightarrow \tilde{\mathcal{J}}$ be the zero section and $\sigma: \tilde{\mathcal{M}}_{g} \longrightarrow \tilde{\mathcal{J}}^{g-1}$ the section which sends $[C, \eta] \mapsto \eta$.

Let $\tilde{\Theta}$ be the line bundle corresponding to the theta divisor on $\tilde{\mathcal{J}}^{g-1}$. Then $\tilde{\Theta}=\delta^{*} \Theta$, and so $\alpha_{*} \mathrm{c}_{1}\left(\tilde{\mathrm{f}}_{g-1 *} \tilde{\Theta}^{\otimes n}\right)=2^{g-1}\left(2^{g}+1\right) \mathrm{c}_{1}\left(\mathrm{f}_{g-1 *} \Theta^{\otimes n}\right)$. Let $\tilde{\lambda}$ be the determinant of the Hodge bundle of the fibration $\tilde{\mathrm{f}}$. Then $\tilde{\lambda}=\alpha^{*} \lambda$, and so $\alpha_{*} \mathrm{c}_{1}(\tilde{\lambda})=$ $2^{g-1}\left(2^{g}+1\right) \mathrm{c}_{1}(\lambda)$. If $\tilde{\mu}:=\sigma^{*} \tilde{\Theta}$, then $\alpha_{*} \mathrm{c}_{1}(\tilde{\mu})=2^{g-2}\left(2^{g}+1\right) \mathrm{c}_{1}(\lambda)$. Let $\tilde{\mathcal{L}}$ be the canonical line bundle on $\tilde{\mathcal{J}}$ of Corollary 4.1. Then $\tilde{\mathcal{L}}=\gamma^{*} \mathcal{L}$. One can see that $c_{1}\left(\tilde{\mathrm{f}}_{*} \phi^{*} \tilde{\Theta}^{\otimes n}\right)=2^{2 g} c_{1}\left(\tilde{\mathrm{f}}_{g-1 *} \tilde{\Theta}^{\otimes n}\right)$. This is an application of the GRR theorem. One can also see that the restrictions of $\phi^{*} \tilde{\Theta}$ and $\tilde{\mathcal{L}}^{\otimes 2}$ on the fibers of the map $\tilde{f}$ are the same. This can be proved by using Proposition 3.5 of Ch. 2 in [5] and Riemann's constant theorem. Therefore, by the see-saw principle, the line bundles $\tilde{\mathcal{L}}^{\otimes 2}$ and $\phi^{*} \tilde{\Theta}$ are isomorphic up to tensor by the pull back of a line bundle from $\tilde{\mathcal{M}}_{g}$. Since $\tilde{s}^{*} \tilde{\mathcal{L}}^{\otimes 2} \cong O$ and $\tilde{s}^{*} \phi^{*} \tilde{\Theta} \cong \tilde{\mu}$, we have $\tilde{\mathcal{L}}^{\otimes 2 n} \otimes \tilde{\mathrm{f}}^{*} \tilde{\mu}^{\otimes n} \cong \phi^{*} \tilde{\Theta}^{\otimes n}$. By applying $\tilde{\mathrm{f}}_{*}$ and taking $\mathrm{c}_{1}$, we have $\mathrm{c}_{1}\left(\tilde{\mathrm{f}}_{*} \tilde{\mathcal{L}}^{\otimes 2 n}\right)+(4 n)^{g} n \mathrm{c}_{1}(\tilde{\mu})=2^{2 g} \mathrm{c}_{1}\left(\tilde{\mathrm{f}}_{g-1} \tilde{\Theta}^{\otimes n}\right)$. Now apply $\alpha_{*}$ to get

$$
\begin{aligned}
& -2^{g-1}(2 n)^{g} 2^{g-1}\left(2^{g}+1\right) \mathrm{c}_{1}(\lambda)+(4 n)^{g} n 2^{g-2}\left(2^{g}+1\right) \mathrm{c}_{1}(\lambda) \\
& \quad=2^{2 g} 2^{g-1}\left(2^{g}+1\right) \mathrm{c}_{1}\left(\mathrm{f}_{g-1} \Theta^{\otimes n}\right) .
\end{aligned}
$$


Therefore $\mathrm{c}_{1}\left(\mathrm{f}_{g-1}{ }^{*} \Theta^{\otimes n}\right)=\frac{1}{2} n^{g}(n-1) \mathrm{c}_{1}(\lambda)$. Since Pic $\mathcal{M}_{g}$ is freely generated by $\lambda$ [1], this concludes the proof of Theorem [C.

4.2. Alternative proof of Theorem $\mathbf{C}$. This is an application of the GRR theorem; see also Appendice 2 in [6] for a similar calculation. We keep the notation of section 4.1. In the above diagram (11), let $\phi$ be the map which sends $L \in \tilde{\mathcal{J}}$, sitting over the point $[C, \eta] \in \tilde{\mathcal{M}}_{g}$, to $L \otimes \eta \in \tilde{\mathcal{J}}^{g-1}$. By Lemma 3.2 we have $\Omega_{\tilde{\mathcal{J}} / \tilde{\mathcal{M}}_{g}} \cong \tilde{\mathrm{f}}^{*} \tilde{s}^{*} \Omega_{\tilde{\mathcal{J}} / \tilde{\mathcal{M}}_{g}}$, and since $\phi$ is an isomorphism, we get

$$
\Omega_{\tilde{\mathcal{J}}^{g-1} / \tilde{\mathcal{M}}_{g}} \cong \tilde{\mathrm{f}}_{g-1}^{*} \sigma^{*} \Omega_{\tilde{\mathcal{J}}^{g-1} / \tilde{\mathcal{M}}_{g}} .
$$

We apply GRR to the fibration $\tilde{\mathrm{f}}_{g-1}: \tilde{\mathcal{J}}^{g-1} \longrightarrow \tilde{\mathcal{M}}_{g}$. It is

$$
\operatorname{ch}\left(\tilde{\mathrm{f}}_{g-1} !\left(\tilde{\Theta}^{\otimes n}\right)\right)=\tilde{\mathrm{f}}_{g-1 *}\left(\operatorname{ch}\left(\tilde{\Theta}^{\otimes n}\right) \cdot \operatorname{td}\left(\Omega_{\tilde{\mathcal{J}}^{g-1} / \tilde{\mathcal{M}}_{g}}^{\vee}\right)\right) .
$$

We get

$$
\mathrm{c}_{1}\left(\tilde{\mathrm{f}}_{g-1 *} \tilde{\Theta}^{\otimes n}\right)=\frac{n^{g+1}}{(g+1) !} \mathrm{f}_{*} \mathrm{c}_{1}^{g+1}(\tilde{\Theta})-\frac{n^{g}}{2} \mathrm{c}_{1}(\tilde{\lambda}) .
$$

The vanishing of the terms on the right hand side containing the "factor" $c_{1}^{k}$, with $k \leq g-1$, in the expansion of $\operatorname{ch}\left(\tilde{\Theta}^{\otimes n}\right)$, is a consequence of the projection formula and the fact that $\Omega_{\tilde{J}^{g-1} / \tilde{\mathcal{M}}_{g}} \cong \tilde{\mathrm{f}}_{g-1}^{*} E$, where $E$ is a vector bundle; see Lemma 3.2 The form of the term containing the "factor" $c_{1}^{g-1}$ is due to the Poincaré formula. The appearance of $\tilde{\lambda}$ is a consequence of Corollary 4.1

Now suppose that, say, $\mathrm{c}_{1}\left(\mathrm{f}_{g-1 *} \Theta^{\otimes n}\right)=a(n) \mathrm{c}_{1}(\lambda)$ and $\mathrm{f}_{g-1 *} \mathrm{c}_{1}^{g+1}(\Theta)=b \mathrm{c}_{1}(\lambda)$, where $a(n), b \in \mathbb{Z} \mathbb{1}$. Then $\mathrm{c}_{1}\left(\tilde{\mathrm{f}}_{g-1 *} \tilde{\Theta}^{\otimes n}\right)=a(n) \mathrm{c}_{1}(\tilde{\lambda})$ and $\tilde{\mathrm{f}}_{g-1 *} \mathrm{c}_{1}^{g+1}(\tilde{\Theta})=b \mathrm{c}_{1}(\tilde{\lambda})$. We get $a(n)=\frac{n^{g+1}}{(g+1) !} b-\frac{n^{g}}{2}$. For $n=1$, the above gives that $b=(g+1) !\left(a(1)+\frac{1}{2}\right)$. But $a(1)=0$, because the line bundle $\tilde{\mathrm{f}}_{g-1 *} \tilde{\Theta}$ has by definition a nowhere zero section, and so it is the trivial bundle. Hence $b=\frac{(g+1) !}{2}$, and so $c_{1}\left(\mathrm{f}_{g-1 *} \Theta^{\otimes n}\right)=$ $\frac{1}{2} n^{g}(n-1) \mathrm{c}_{1}(\lambda)$.

\section{REFERENCES}

1. Arbarello E. and Cornalba M.: The Picard group of the moduli spaces of curves. Topology 26, 152-171 (1987). MR 88e:14032

2. Barth W., Peters C. and van de Ven A.: Compact Complex Surfaces. Springer-Verlag, 1984. MR 86c:32026

3. Brasch H-J.: Lifting level D-structures of abelian varieties. Arch. Math. 60, 553-562 (1993). MR 94b:14038

4. Faltings G. and Chai Ch-L.: Degeneration of Abelian Varieties. Springer-Verlag, 1990. MR 92d:14036

5. Lange H. and Birkenhake Ch.: Complex Abelian Varieties. Springer-Verlag, 1992. MR 94j:14001

6. Moret-Bailly, L.: Pinceaux de variétés abéliennes. Astérisque 129 (1985). MR 87j:14069

7. Mumford D.: Tata Lectures on Theta I. Progress in Mathematics, vol. 28, Birkhäuser, 1983. MR 85h:14026

8. Teixidor i Bigas M.: Half-canonical series on algebraic curves. Transactions of A.M.S. 302, 99-115 (1987). MR 88e:14037

9. Teixidor i Bigas M.: The divisor of curves with a vanishing theta-null. Compositio Math. 66, 15-22 (1988). MR 89c:14040

Department of Mathematics, University of Crete, 71409, Heraklion-Crete, Greece E-mail address: kouvid@math.uch.gr 